\title{
Prediction of Congestive Heart Failure in Patients with Non Valvular Atrial Fibrillation
}

\author{
Koichiro Imai, Hiroyuki Okura, Tomoko Tamada, Kenzo Fukuhara, Terumasa Koyama, \\ Teruyoshi Kume, Akihiro Hayashida, Takahiro Kawamoto, Yoji Neishi and Kiyoshi Yoshida
}

\begin{abstract}
Objective Patients with atrial fibrillation (AF) have an increased risk of congestive heart failure (CHF) as well as ischemic stroke. The aim of this study was to investigate the clinical predictors of CHF in patients with non-valvular AF (NVAF).

Methods Three hundred and forty-seven patients (derivation cohort) with NVAF were retrospectively evaluated between 2004 and 2005. The associations between potential risk factors and CHF were tested using a Cox proportional hazards analysis, and a risk score for predicting CHF was created. The model was then validated in 161 patients (validation cohort) enrolled between 2008 and 2010. During the follow-up period, 41 patients in the derivation cohort developed CHF requiring hospitalization due to New York Heart Association (NYHA) class III or IV disease. Four independent risk factors were identified, each of which was assigned a number of points as follows: Age $\geq 72$ years ( 1 point), heart rate $\geq 80 \mathrm{bpm}$ ( 1 point), hypertension (1 point), and a previous history of congestive heart failure ( 2 points). The patients were grouped into one of three risk categories according to the calculated risk score $\left(\mathrm{ARC}_{2} \mathrm{H}\right.$ score): low risk (0 points), intermediate risk (1-3 points) and high risk (4-5 points).

Result In the derivation cohort, the annual rates of CHF in these risk categories were $0 \%, 2.5 \%$ and $18 \%$ per year respectively. In the validation cohort, the corresponding rates were $0.8 \%, 8 \%$ and $35 \%$ per year respectively.

Conclusion A simple clinical risk score, the $\mathrm{ARC}_{2} \mathrm{H}$ score, was developed to predict $\mathrm{CHF}$ in patients with NVAF and validated in an independent cohort.
\end{abstract}

Key words: atrial fibrillation, $\mathrm{ARC}_{2} \mathrm{H}$ score, congestive heart failure

(Intern Med 53: 7-12, 2014)

(DOI: 10.2169/internalmedicine.53.0962)

\section{Introduction}

Atrial fibrillation (AF) is the most common sustained cardiac arrhythmia. It is well recognized that stroke and congestive heart failure (CHF) are the two most important clinical comorbid conditions associated with AF. It has been reported that the annual rate of stroke in AF patients ranges from less than $2 \%$ to more than $10 \%$ per year (1-6) depending on the risk of stroke (7). On the other hand, the incidence of $\mathrm{CHF}$ among $\mathrm{AF}$ patients varies from $1.7 \%$ to more than $10 \%$ per year (8-10), depending on the patient's background clinical condition.
Although a previous report suggested that CHF has an adverse impact on the prognosis of patients with AF independent of other cardiovascular diagnoses and risk factors (9), other reports have suggested that AF is not associated with worse mortality in patients with advanced heart failure $(11,12)$. These discordant results suggest that the risk of CHF differ among AF patient with different clinical backgrounds. Therefore, it is especially important to assess the risk of $\mathrm{CHF}$ and optimally manage patients with $\mathrm{AF}$ who are at a high likelihood of developing CHF.

The $\mathrm{CHADS}_{2}$ score has been established to be a simple and reproducible risk score for predicting stroke in patients with AF (13). Although AF patients have an increased risk 
Table 1. Clinical Characteristics of the Derivative Cohort

\begin{tabular}{lccc}
\hline & $\begin{array}{c}\text { CHF } \\
(\mathrm{n}=41)\end{array}$ & $\begin{array}{c}\text { Non-CHF } \\
(\mathrm{n}=306)\end{array}$ & p value \\
\hline Age, years & $73.9 \pm 8.9$ & $71.1 \pm 9.1$ & 0.038 \\
Female gender, n (\%) & $19(46)$ & $102(29)$ & 0.025 \\
Body mass index, kg/m² & $22.6 \pm 3.1$ & $23.9 \pm 4.1$ & 0.193 \\
Heart rate, beats/min & $85.7 \pm 18.4$ & $79.4 \pm 17.6$ & 0.018 \\
Hypertension, n (\%) & $30(73)$ & $156(50)$ & 0.021 \\
Hyperlipidemia, n (\%) & $10(24)$ & $80(26)$ & 0.222 \\
Diabetes mellitus, n (\%) & $10(24)$ & $70(23)$ & 0.393 \\
Smoking, n (\%) & $18(43)$ & $104(33)$ & 0.108 \\
History of IHD, n (\%) & $5(12)$ & $18(6)$ & 0.194 \\
History of stroke, $\mathrm{n}(\%)$ & $5(12)$ & $31(10)$ & 0.562 \\
History of CHF, n (\%) & $30(73)$ & $59(20)$ & $<0.0001$ \\
\hline
\end{tabular}

CHF: congestive heart failure, IHD: ischemic heart disease

of $\mathrm{CHF}$, no risk score for predicting $\mathrm{CHF}$ in patients with AF has been established. Therefore, the purpose of this study was to investigate predictors of CHF in patients with $\mathrm{AF}$ and to propose a clinical score for predicting $\mathrm{CHF}$ in patients with non valvular AF (NVAF).

\section{Materials and Methods}

\section{Study population}

Between October 2004 and December 2005, 592 (3.2\%) patients with $\mathrm{AF}$ were identified and included from a retrospective database of 18,568 consecutive patients who underwent 12-lead electrocardiogram (ECG) examination at our hospital. Patients with paroxysmal AF, pacemaker implantation or valvular $\mathrm{AF}$ were excluded. In addition, patients were also excluded if their clinical outcomes could not be evaluated. Ultimately, 347 patients (derivation cohort: 121 women, mean age $71 \pm 9$ years, range: 18 to 93 years) with (NVAF) were enrolled. The patients were divided into two groups: patients who developed $\mathrm{CHF}$ requiring hospitalization due to New York Heart Association (NYHA) class III or IV disease (CHF group, $\mathrm{n}=41$ ) and those who did not develop CHF (non-CHF group, $n=306$ ) during the follow-up period (median: 42 months). CHF was defined according to the Framingham Heart Study criteria (14). To identify predictors of CHF, clinical characteristics, laboratory data and medications were compared between the two groups. Based on the results obtained from the derivation cohort, a clinical risk score to predict CHF was constructed. The constructed model was then validated in a validation cohort. A total of 486 consecutive outpatients with newly diagnosed NVAF who visited our hospital between 2008 and 2010 were identified. Patients with paroxysmal AF or pacemaker implantation were excluded. Ultimately, 161 patients (validation cohort: 51 women, mean age: $71 \pm 2$ years, range: 36 to 98 years, mean follow-up: 24 months) were enrolled.

\section{Criteria for persistent atrial fibrillation}

AF was defined according to the ACC/AHA/ESC guide-
Table 2. Medications of the Derivative Cohort

\begin{tabular}{lrrr}
\hline & \multicolumn{1}{c}{ CHF } \\
& \multicolumn{1}{c}{$\begin{array}{c}\text { Non-CHF } \\
(\mathrm{n}=306)\end{array}$} & p value \\
\hline ACE-I / ARB, n (\%) & $20(48)$ & $124(41)$ & 0.314 \\
CCB, n (\%) & $16(39)$ & $90(29)$ & 0.210 \\
Beta-blockers, n (\%) & $10(24)$ & $54(18)$ & 0.296 \\
Digitalis, n (\%) & $18(43)$ & $130(42)$ & 0.427 \\
Warfarin, n (\%) & $22(53)$ & $179(58)$ & 0.556 \\
Statins, n (\%) & $6(14)$ & $40(13)$ & 0.182 \\
\hline
\end{tabular}

ACE-I:angiotensin-converting enzyme inhibitors, ARB: angiotensin II receptor blockers, CCB:calcium channel blockers

Table 3. Univariable and Multivariable Predictors of CHF

\begin{tabular}{lccccr}
\hline & \multicolumn{2}{c}{ Univariable } & & \multicolumn{2}{c}{ Multivariable } \\
\hline & HR $(95 \% \mathrm{CI})$ & $\mathrm{p}$ value & & HR $(95 \% \mathrm{CI})$ & $\mathrm{p}$ value \\
\cline { 2 - 3 } \cline { 5 - 6 } History of CHF & $6.29(3.65-10.8)$ & $<0.0001$ & & $6.44(3.62-11.5)$ & $<0.0001$ \\
Heart rate, bpm & $1.12(1.02-1.34)$ & 0.020 & & $1.03(1.00-1.06)$ & 0.035 \\
Age, years & $1.86(1.16-2.99)$ & $<0.01$ & & $1.92(1.13-3.29)$ & 0.016 \\
Hypertension & $2.69(1.65-4.40)$ & $<0.01$ & & $2.30(1.34-3.90)$ & 0.023 \\
Female & $1.87(1.03-3.42)$ & 0.042 & & $0.68(0.37-1.23)$ & 0.205 \\
\hline
\end{tabular}

CHF: congestive heart failure, HR:hazard ratio, $\mathrm{CI}$ :confidence interval

bpm: beats per minutes

lines (12). The presence of AF was confirmed based on the results of two consecutive 12-lead ECG examinations performed at least seven days apart. All study patients were diagnosed as presenting with $\mathrm{AF}$ for at least one year or more at the time of study entry and exhibited AF that persisted throughout the follow-up period. Heart rate [(HR, beat/min (bpm)] at rest was defined as the average of 10 consecutive beats during resting 12-lead ECG.

The study protocol was approved by the Institutional Review Committee on Human Research at our institution.

\section{Statistical analysis}

The data are expressed as the mean $\pm \mathrm{SD}$. The two groups were compared using unpaired Student's $t$-test and chi-square test. A Cox proportional hazard analysis was used to identify the independent risk factors for CHF in the derivation cohort. A receiver operator characteristic (ROC) curve analysis was used to determine the cut-off values of age and HR. All statistical analyses were performed using the SAS software program, version 8.2 (SAS Institute, Cary, NC, USA).

\section{Results}

During the follow-up period (median: 42 months), 41 (12\%) patients in the derivation cohort developed CHF requiring hospitalization.

Table 1 summarizes the baseline clinical characteristics of the two groups in the derivation cohort. Age and HR were significantly higher in the CHF group than in the non-CHF group. A female gender, hypertension, history of CHF and history of open heart surgery were more frequently observed in the CHF group than in the non-CHF group. The medica- 


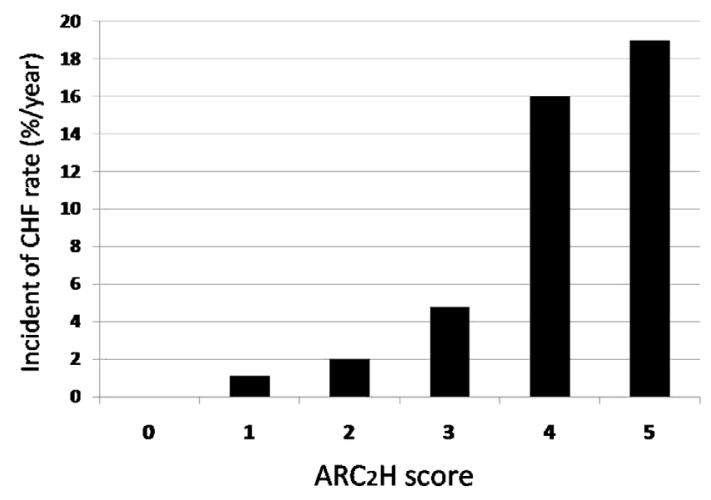

Figure 1. Relationship between the $\mathrm{ARC}_{2} \mathrm{H}$ score and incidence of $\mathrm{CHF}$ in patients with the derivation cohort. As the $\mathrm{ARC}_{2} \mathrm{H}$ score increased, incidence of $\mathrm{CHF}$ increased.

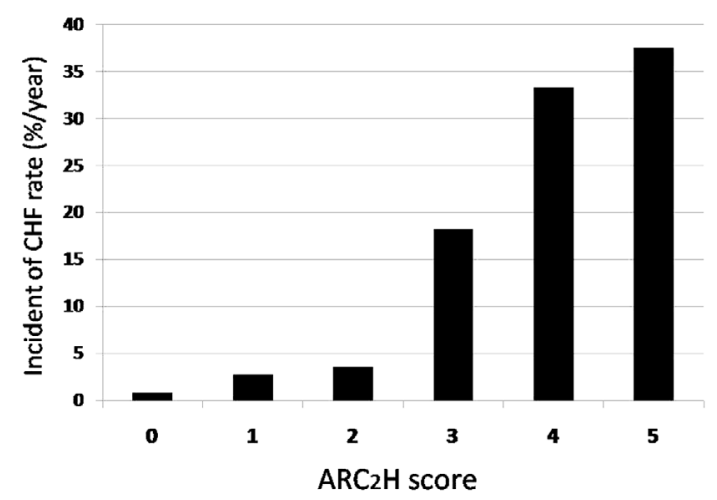

Figure 3. Relationship between the $\mathrm{ARC}_{2} \mathrm{H}$ score and incidence of $\mathrm{CHF}$ in patients with the validation cohort. As the $\mathrm{ARC}_{2} \mathrm{H}$ score was increased, the incidence of was $\mathrm{CHF}$ increased.

tions are summarized in Table 2. There were no significant differences in medications between the two groups.

The univariable predictors of CHF were a history of CHF, age, female gender, hypertension and HR. According to the multivariable Cox proportional hazard analysis, age, hypertension, HR and a history of CHF were independent predictors of CHF (Table 3). A cut-off value of an age $\geq 72$ years was identified in the ROC curve analysis. Similarly, according to the ROC curve analysis, HR $\geq 80 \mathrm{bpm}$ was identified as a cut-off value predicting $\mathrm{CHF}$.

\section{Risk score for predicting CHF in patients with NVAF}

Based on the results obtained in the derivation cohort, we constructed a clinical risk score, the " $\mathrm{ARC}_{2} \mathrm{H}$ " (Age, HR, history of $\mathrm{CHF}$ and Hypertension) score, to predict $\mathrm{CHF}$ in patients with NVAF. The $\mathrm{ARC}_{2} \mathrm{H}$ score was calculated by assigning 1 point each for the presence of hypertension, an age $\geq 72$ years and $\mathrm{HR} \geq 80 \mathrm{bpm}$ and 2 points to a history of CHF because a history of CHF added significant independent predictive power to the other clinical risk factors. Fig. 1 shows the relationship between the $\mathrm{ARC}_{2} \mathrm{H}$ score and the rate of $\mathrm{CHF}$ per year in the derivation cohort. The rate of $\mathrm{CHF}$ increased as the $\mathrm{ARC}_{2} \mathrm{H}$ score increased. The patients

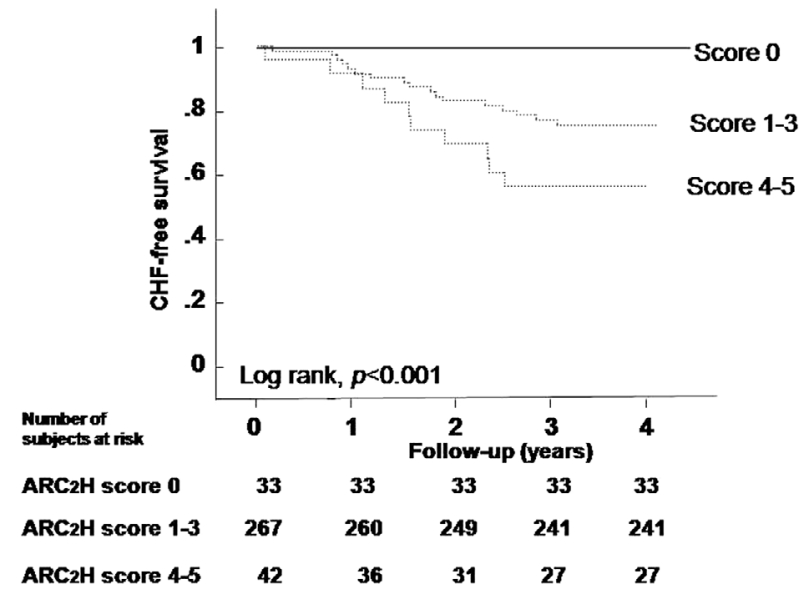

Figure 2. Kaplan-Meier plot of the CHF-free survival curves in each risk category of the derivation cohort.CHF-free survival rate was significantly lower in high risk category group than in intermediate and low risk category groups.

Table 4. Clinical Characteristics and Medication of the Validation Cohort

\begin{tabular}{lr}
\hline & $\mathrm{n}=161$ \\
\hline Age, years & $71.2 \pm 2.0$ \\
Female gender, $\mathrm{n}(\%)$ & $51(31)$ \\
Body mass index, kg/m² & $23.6 \pm 2.1$ \\
Heart rate, bpm & $80.1 \pm 17.7$ \\
Hypertension, n (\%) & $87(54)$ \\
Hyperlipidemia, n (\%) & $87(54)$ \\
Diabetes mellitus, n (\%) & $32(20)$ \\
Smoking, $\mathrm{n}(\%)$ & $20(13)$ \\
History of IHD, n (\%) & $9(6)$ \\
History of stroke, n (\%) & $18(11)$ \\
History of CHF, n (\%) & $24(23)$ \\
Medications, n (\%) & \\
ACE-I / ARB, n (\%) & $93(58)$ \\
CCB, n (\%) & $57(35)$ \\
Beta-blockers, n (\%) & $59(37)$ \\
Digitalis, n (\%) & $45(28)$ \\
Warfarin, n (\%) & $21(13)$ \\
Statins, $\mathrm{n}(\%)$ & $(88)$ \\
\hline
\end{tabular}

CHF, congestive heart failure, IHD: ischemic heart disease, ACE-I: angiotensin-converting enzyme inhibitors, ARB: angiotensin II receptor blockers, CCB: calcium channel blockers, bpm: beats per minutes

were then grouped into one of the three risk categories: low risk ( 0 points), intermediate risk (1 to 3 points) and high risk (4 to 5 points). In the derivation cohort, the annual rates of $\mathrm{CHF}$ in the risk categories were $0 \%$ (low risk), $2.5 \%$ (intermediate risk), and $18 \%$ (high risk) per year, respectively. Fig. 2 shows a Kaplan-Meier plot of the CHF-free survival curves for each risk category in the derivation cohort. The patients in the high-risk category had a significantly lower CHF-free survival rate.

Table 4 summarizes the clinical characteristics and medications of the validation cohort. Digitalis was less frequently used in the validation cohort than in the derivation cohort. On the other hand, beta-blockers and warfarin were more 

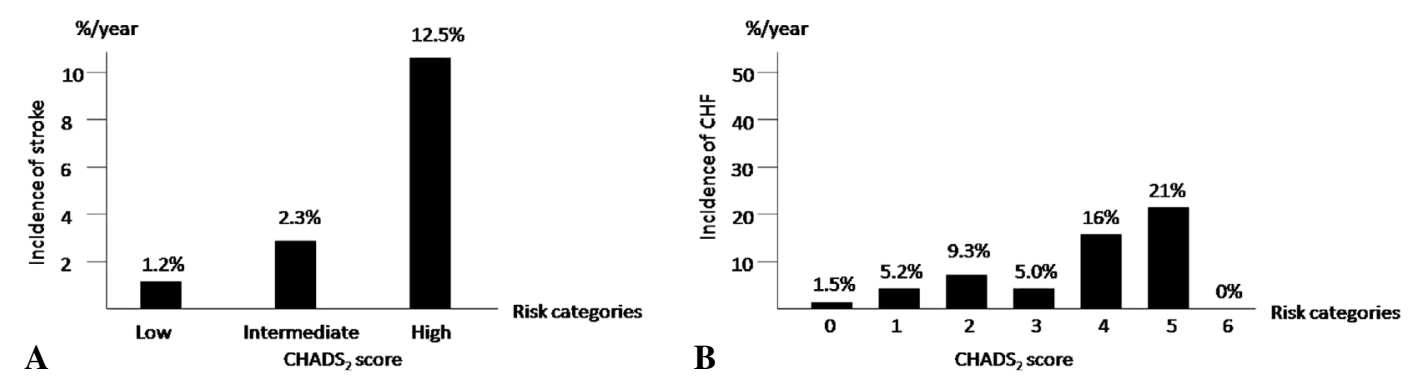

Figure 4. A: Relationship between the CHADS 2 score and incidence of stroke in study patients. Annual rate of stroke was strongly related to the stroke risk categories. B: Relationship between the $\mathrm{CHADS}_{2}$ score and incidence of $\mathrm{CHF}$ in study patients. Annual rate of $\mathrm{CHF}$ did not differ between the stroke risk categories.

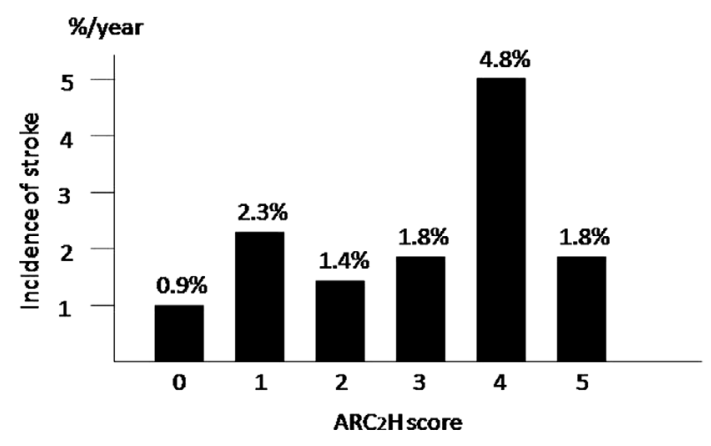

Figure 5. Relationship between the $\mathrm{ARC}_{2} \mathrm{H}$ score and incidence of stroke in study patients. Annual risk of stroke did not differ between the risk categories stratified by the $\mathrm{ARC}_{2} \mathrm{H}$ score.

frequently prescribed in the validation cohort than in the derivation cohort.

Fig. 3 shows the annual rate of $\mathrm{CHF}$ in the validation group. Similar to that observed in the derivation group, the rate of $\mathrm{CHF}$ increased as the $\mathrm{ARC}_{2} \mathrm{H}$ score increased. In the validation cohort, the annual rates of $\mathrm{CHF}$ in the risk categories were $0.8 \%$ (low risk), $8 \%$ (intermediate risk) and $35 \%$ (high risk) per year, respectively.

Both the derivation and validation cohorts were grouped according to the stroke risk categories (low risk, intermediate risk and high risk) stratified by the $\mathrm{CHADS}_{2}$ score. As expected, the annual rate of stroke was strongly related to the stroke risk categories (Fig. 4A). On the other hand, the annual rate of $\mathrm{CHF}$ did not differ between the stroke risk categories (Fig. 4B). Conversely, annual risk of stroke did not differ between the risk categories stratified by the $\mathrm{ARC}_{2} \mathrm{H}$ score (Fig. 5).

The derivation cohort was further divided into two groups according to the presence or absence of a previous history of CHF. Among the patients with a history of CHF, the independent predictors of $\mathrm{CHF}$ were hypertension $(\mathrm{OR}=3.43$, 95\% CI: $1.19-9.82, \mathrm{p}=0.022)$ and $\mathrm{HR}(\mathrm{OR}=1.03$, 95\% CI: $1.00-1.06, \mathrm{p}=0.045)$. On the other hand, only hypertension $(\mathrm{OR}=2.08,95 \% \mathrm{CI}: 1.10-3.94, \mathrm{p}=0.024)$ and age $(\mathrm{OR}=1.05$, 95\% CI: $1.01-1.09, \mathrm{p}=0.015)$ were independent predictors of $\mathrm{CHF}$ in the NVAF patients without a history of CHF.

Discussion

Our present study showed that age, hypertension, HR and a previous history of $\mathrm{CHF}$ were independent predictors of $\mathrm{CHF}$ in patients with NVAF. The $\mathrm{ARC}_{2} \mathrm{H}$ score derived from these independent predictors may be used as a simple and useful clinical score to assess the risk of CHF in patients with NVAF.

It has been reported that a previous history of CHF is an independent predictor of future CHF (13). Our results showing that a history of $\mathrm{CHF}$ added significant independent predictive power to the other clinical risk factors are concordant with the findings of previous reports. On the other hand, the predictive role of baseline HR has not yet been established. Although the current ACC/AHA/ESC guidelines recommend a target $\mathrm{HR}$ of 60 to $80 \mathrm{bpm}$ at rest in $\mathrm{AF}$ patients who receive rate control (15), the relationship between $\mathrm{HR}$ and the risk of $\mathrm{CHF}$ in AF patients remains controversial. Groenveld et al. reported that there are no significant differences in cardiovascular mortality or morbidity between AF patients with a HR of $\geq 80 \mathrm{bpm}$ or $\mathrm{HR}<80 \mathrm{bpm}$ (16). On the other hand, Reinstra et al. reported that a lower HR $(<80 \mathrm{bpm})$ is associated with a poorer prognosis (17). Similarly, Castagnon et al. reported that the resting heart rate is only an important predictor of the outcome in patients with stable chronic heart failure without AF (18). More recently, Van Gelder et al. reported that there are no significant differences in cardiovascular mortality or morbidity between patients treated with strict rate control $(\mathrm{HR}<80 \mathrm{bpm})$ and lenient rate control $(\mathrm{HR}<110 \mathrm{bpm})$ strategies (19). Therefore, it remains unknown if strict rate control will prevent $\mathrm{CHF}$ and improve the prognosis in our study population.

It is well known that hypertension and age are independent risk factors for both $\mathrm{AF}$ and $\mathrm{CHF}(8,14)$. Therefore, our results showing that age and hypertension are independent predictors of $\mathrm{CHF}$ are also concordant with the findings of these previous reports $(8,14)$.

It has been reported that elderly women are predominantly observed among patients with heart failure with a preserved ejection fraction (20-22). This phenomenon may be related to gender differences in the age-related deteriora- 
tion of the diastolic function (23). In the present study, a female gender was a univariable predictor of CHF but not an independent predictor of $\mathrm{CHF}$ in the multivariable analysis. Differences in the study population and the small sample size of this study may explain the lack of association between gender and CHF. The rate of hospitalization for $\mathrm{CHF}$ was slightly different between the derivation and validation cohorts in our study. The left ventricular ejection fraction (LVEF) was significantly lower in the validation cohort than in the derivation cohort (LVEF; $54 \pm 11.9 \%$ vs. $60 \pm 10.8$, p < 0.036). Therefore, it is possible that differences in the baseline cardiac function affected the difference in the hospitalization rate observed between the derivation and validation groups.

Although the CHADS 2 score (13) is a predictor of stroke, it was not related to the incidence of $\mathrm{CHF}$ in our study population. Conversely, the $\mathrm{ARC}_{2} \mathrm{H}$ score was not related to the incidence of stroke. Therefore, the $\mathrm{ARC}_{2} \mathrm{H}$ score and the $\mathrm{CHADS}_{2}$ score may be used to assess the risk of either CHF or stroke independently in patients with NVAF. A previous study reported that the echo Doppler derived index of the left ventricular filling pressure, E/e', predicts mortality and hospitalization due to CHF (24). Therefore, a clinical score may be used as a screening tool to select NVAF patients who should be referred for echocardiography examinations.

Suzuki et al. reported that the H2ARDD (heart diseases $=2$ points, anemia $=1$ point, renal dysfunction $=1$ point, diabetes $=$ 1 point and diuretic use $=1$ point; range 0 to 6 points) score predicts $\mathrm{CHF}$ in patients with $\mathrm{AF}$ (25). In our study, there were no significant differences in the rates of renal dysfunction, diuretic use, anemia or diabetes between the CHF and non-CHF groups. This difference between their study and our study may in be explained in part by the differences in the study populations. Their study included patients with valvular heart disease, while our study enrolled patients with NVAF. In addition, their cannot be denied until they perform a validation study.

In AF patients, antithrombotic therapy using warfarin or dabigatran has been established as a standard therapy for the preventing of strokes in patients with an $\geq$ intermediate stroke risk according to the $\mathrm{CHADS}_{2}$ score $(26,27)$. On the other hand, specific medications to lower the risk of $\mathrm{CHF}$ have not yet been established. Previous studies have reported that angiotensin-converting-enzyme inhibitors, betablockers and angiotensin II receptor blockers may improve the outcomes of patients with CHF with or without a preserved left ventricular ejection fraction (28-31).

Recently, it was reported that irbesartan, an angiotensin II receptor blocker, may decrease the rate of hospitalization due to heart failure in patients with AF (32). Therefore, the impact of medical interventions on the risk of $\mathrm{CHF}$ requires further investigation.

\section{Clinical implications}

$\mathrm{AF}$ is an important clinical condition due to both its strong relationship with stroke and its function as a cause of
CHF. The $\mathrm{ARC}_{2} \mathrm{H}$ score simply predicts the risk of $\mathrm{CHF}$, as does the $\mathrm{CHADS}_{2}$ score predict the risk of stroke, in patients with AF. Although echocardiographic indices as well as biomarkers, such as the level of BNP, can be used to further stratify AF patients with a higher risk for CHF, these indices were not included in our present study. The most important clinical implication of this study is that we can predict the risk of CHF using a medical interview and vital signs only, even in the outpatient clinic. Performing risk stratification of patients with NVAF using the $\mathrm{ARC}_{2} \mathrm{H}$ score is useful for identifying high-risk patients who require aggressive medical therapy.

\section{Study limitations}

First, although consecutive patients were enrolled, this study may be weakened by the fact that it was a small, retrospective, single-center study. Therefore, the results must be confirmed in a large, prospective, multicenter studies. Second, the median follow-up period was only 42 months in the derivation group. This relatively short follow-up duration may have resulted in the underestimation of the true number of CHF events. Third, patients with paroxysmal AF were excluded. Therefore, our results cannot be generalized to all patients with AF. Finally, the impact of medical treatments on modifying the risk of CHF remains unknown and should be investigated further.

\section{Conclusion}

Age, heart rate, hypertension and a history of CHF are independently associated with $\mathrm{CHF}$ in patients with NVAF. The $\mathrm{ARC}_{2} \mathrm{H}$ score may be used as a simple clinical score for predicting $\mathrm{CHF}$ in patients with NVAF.

The authors state that they have no Conflict of Interest (COI).

\section{References}

1. Kopecky SL, Gersh BJ, McGoon MD, et al. Lone atrial fibrillation in elderly persons: a marker for cardiovascular risk. Arch Intern Med 159: 1118-1122, 1999.

2. Risk factors for stroke and efficacy of antithrombotic therapy in atrial fibrillation. Analysis of pooled data from five randomized controlled trials. Arch Intern Med 154: 1449-1457, 1994.

3. Patients with nonvalvular atrial fibrillation at low risk of stroke during treatment with aspirin: Stroke Prevention in Atrial Fibrillation III Study. The SPAF III Writing Committee for the Stroke Prevention in Atrial Fibrillation Investigators. JAMA 279: 12731277, 1998.

4. Predictors of thromboembolism in atrial fibrillation: I. Clinical features of patients at risk. The Stroke Prevention in Atrial Fibrillation Investigators. Ann Intern Med 116: 1-5, 1992.

5. van Latum JC, Koudstaal PJ, Venables GS, van Gijn J, Kappelle LJ, Algra A. Predictors of major vascular events in patients with a transient ischemic attack or minor ischemic stroke and with nonrheumatic atrial fibrillation. European Atrial Fibrillation Trial (EAFT) Study Group. Stroke 26: 801-806, 1995.

6. Van Gelder IC, Hagens VE, Bosker HA, et al. A comparison of rate control and rhythm control in patients with recurrent persis- 
tent atrial fibrillation. N Engl J Med 347: 1834-1840, 2002.

7. Wang TJ, Larson MG, Levy D, et al. Temporal relations of atrial fibrillation and congestive heart failure and their joint influence on mortality: the Framingham Heart Study. Circulation 107: 2920$2925,2003$.

8. Rationale and design of a study assessing treatment strategies of atrial fibrillation in patients with heart failure: the Atrial Fibrillation and Congestive Heart Failure (AF-CHF) trial. Am Heart J 144: 597-607, 2002.

9. Olsson LG, Swedberg K, Ducharme A, et al. Atrial fibrillation and risk of clinical events in chronic heart failure with and without left ventricular systolic dysfunction: results from the Candesartan in Heart failure-Assessment of Reduction in Mortality and morbidity (CHARM) program. J Am Coll Cardiol 47: 1997-2004, 2006.

10. McKee PA, Castelli WP, McNamara PM, Kannel WB. The natural history of congestive heart failure: the Framingham study. N Engl J Med 285: 1441-1446, 1971.

11. Mahoney P, Kimmel S, DeNofrio D, Wahl P, Loh E. Prognostic significance of atrial fibrillation in patients at a tertiary medical center referred for heart transplantation because of severe heart failure. Am J Cardiol 83: 1544-1547, 1999.

12. Crijns HJ, Tjeerdsma G, de Kam PJ, et al. Prognostic value of the presence and development of atrial fibrillation in patients with advanced chronic heart failure. Eur Heart J 21: 1238-1245, 2000.

13. Gage BF, Waterman AD, Shannon W, Boechler M, Rich MW, Radford MJ. Validation of clinical classification schemes for predicting stroke: results from the National Registry of Atrial Fibrillation. JAMA 285: 2864-2870, 2001.

14. Benjamin EJ, Levy D, Vaziri SM, D'Agostino RB, Belanger AJ, Wolf PA. Independent risk factors for atrial fibrillation in a population-based cohort. The Framingham Heart Study. JAMA 271: 840-844, 1994.

15. Fuster V, Ryden LE, Asinger RW, et al. ACC/AHA/ESC guidelines for the management of patients with atrial fibrillation. A report of the American College of Cardiology/American Heart Association Task Force on Practice Guidelines and the European Society of Cardiology Committee for Practice Guidelines and Policy Conferences (Committee to develop guidelines for the management of patients with atrial fibrillation) developed in collaboration with the North American Society of Pacing and Electrophysiology. Eur Heart J 22: 1852-1923, 2001.

16. Groenveld HF, Crijns HJ, Rienstra M, Van den Berg MP, Van Veldhuisen DJ, Van Gelder IC. Does intensity of rate control influence outcome in persistent atrial fibrillation? Data of the RACE study. Am Heart J 158: 785-791, 2009.

17. Rienstra M, Van Gelder IC, Van den Berg MP, Boomsma F, Hillege HL, Van Veldhuisen DJ. A comparison of low versus high heart rate in patients with atrial fibrillation and advanced chronic heart failure: effects on clinical profile, neurohormones and survival. Int J Cardiol 109: 95-100, 2006.

18. Castagno D, Skali H, Takeuchi M, et al. Association of heart rate and outcomes in a broad spectrum of patients with chronic heart failure: results from the CHARM (Candesartan in Heart Failure: Assessment of Reduction in Mortality and morbidity) program. J
Am Coll Cardiol 59: 1785-1795, 2012.

19. Van Gelder IC, Groenveld HF, Crijns HJ, et al; RACE II Investigators. Lenient versus strict rate control in patients with atrial fibrillation. N Eng J Med 362: 1363-1373, 2010.

20. Klapholz M, Maurer M, Lowe AM, et al. Hospitalization for heart failure in the presence of a normal left ventricular ejection fraction: results of the New York Heart Failure Registry. J Am Coll Cardiol 43: 1432-1438, 2004.

21. Yancy CW, Lopatin M, Stevenson LW, De Marco T, Fonarow GC. Clinical presentation, management, and in-hospital outcomes of patients admitted with acute decompensated heart failure with preserved systolic function: a report from the Acute Decompensated Heart Failure National Registry (ADHERE) Database. J Am Coll Cardiol 47: 76-84, 2006.

22. Masoudi FA, Havranek EP, Smith G, et al. Gender, age, and heart failure with preserved left ventricular systolic function. J Am Coll Cardiol 41: 217-223, 2003.

23. Okura H, Takada Y, Yamabe A, et al. Age- and gender-specific changes in the left ventricular relaxation: a Doppler echocardiographic study in healthy individuals. Circ Cardiovasc Imaging 2: 41-46, 2009.

24. Okura H, Takada Y, Kubo T, et al. Tissue Doppler-derived index of left ventricular filling pressure, E/E', predicts survival of patients with non-valvular atrial fibrillation. Heart 92: 1248-1252, 2006.

25. Suzuki S, Sagara K, Otsuka T, et al. A new scoring system for evaluating the risk of heart failure events in Japanese patients with atrial fibrillation. Am J Cardiol 110: 678-682, 2012.

26. Adjusted-dose warfarin versus low-intensity, fixed-dose warfarin plus aspirin for high-risk patients with atrial fibrillation: Stroke Prevention in Atrial Fibrillation III randomised clinical trial. Lancet 348: 633-638, 1996.

27. Connolly SJ, Ezekowitz MD, Yusuf S, et al; RE-LY Steering Committee and Investigators. Dabigatran versus warfarin in patients with atrial fibrillation. N Engl J Med 361: 1139-1151, 2009.

28. Yusuf S, Pfeffer MA, Swedberg K, et al. Effects of candesartan in patients with chronic heart failure and preserved left-ventricular ejection fraction: the CHARM-Preserved Trial. Lancet 362: 777781, 2003.

29. Pitt B, Remme W, Zannad F, et al. Eplerenone, a selective aldosterone blocker, in patients with left ventricular dysfunction after myocardial infarction. N Engl J Med 348: 1309-1321, 2003.

30. Granger CB, McMurray JJ, Yusuf S, et al. Effects of candesartan in patients with chronic heart failure and reduced left-ventricular systolic function intolerant to angiotensin-converting-enzyme inhibitors: the CHARM-Alternative trial. Lancet 362: 772-776, 2003.

31. Effect of enalapril on survival in patients with reduced left ventricular ejection fractions and congestive heart failure. The SOLVD Investigators. N Engl J Med 325: 293-302, 1991.

32. ACTIVE I Investigators. Yusuf S, Heale JS, Pogue J, et al. Irbesartan in patients with atrial fibrillation. N Engl J Med 364: 928938, 2011.

(C) 2014 The Japanese Society of Internal Medicine

http://www.naika.or.jp/imonline/index.html 